\title{
Sleep and Second-Language Acquisition Revisited: The Role of Sleep Spindles and Rapid Eye Movements
}

\author{
Kristen Thompson' \\ Aaron Gibbings ${ }^{1,2}$ \\ James Shaw' \\ Laura Ray' \\ Gilles Hébert ${ }^{1}$ \\ Joseph De Koninck (1) ${ }^{1,3}$ \\ Stuart Fogel ${ }^{1-3}$ \\ 'School of Psychology, University of \\ Ottawa, Ottawa, ON, Canada; ${ }^{2}$ Sleep \\ Unit, University of Ottawa Institute of \\ Mental Health at the Royal, Ottawa, ON, \\ Canada; ${ }^{3}$ University of Ottawa Brain and \\ Mind Research Institute, University of \\ Ottawa, Ottawa, ON, Canada
}

\begin{abstract}
Introduction: Second-language learning (SLL) depends on distinct functionalneuroanatomical systems including procedural and declarative long-term memory. Characteristic features of rapid eye movement (REM) and non-REM sleep such as rapid eye movements and sleep spindles are electrophysiological markers of cognitively complex procedural and declarative memory consolidation, respectively. In adults, grammatical learning depends at first on declarative memory ("early SLL") then shifts to procedural memory with experience ("late SLL"). However, it is unknown if the shift from declarative to procedural memory in early vs late SLL is supported by sleep. Here, we hypothesized that increases in sleep spindle characteristics would be associated with early SLL, whereas increases in REM activity (eg, density and EEG theta-band activity time-locked to rapid eye movements) would be associated with late SLL.
\end{abstract}

Methods: Eight Anglophone (English first language) participants completed four polysomnographic recordings throughout an intensive 6-week French immersion course. Sleep spindle data and electroencephalographic spectral power time-locked to rapid eye movements were extracted from parietal temporal electrodes.

Results: As predicted, improvements in French proficiency were associated with changes in spindles during early SLL. Furthermore, we observed increased event-related theta power timelocked to rapid eye movements during late SLL compared with early SLL. The increases in theta power were significantly correlated with improvements in French proficiency.

Discussion: This supports the notion that sleep spindles are involved in early SLL when grammar depends on declarative memory, whereas cortical theta activity time-locked to rapid eye movements is involved in late SLL when grammar depends on procedural memory.

Keywords: memory consolidation, procedural memory, declarative memory, learning, theta, language

\section{Introduction}

The ability to acquire a second language depends on memory systems adapted to support language learning. ${ }^{1}$ Sleep plays an important role in the formation of newly acquired memory for both declarative (eg, memory for facts, figures and events; ie, "knowing what") and procedural (eg, memory for skills, strategy and grammatical rules; ie, "knowing how") memory. Sleep is an opportune time for both passive and active processes that support memory consolidation to occur without interference. ${ }^{2}$ The Dual Process hypothesis ${ }^{2,3}$ and the Active System Consolidation hypothesis ${ }^{4}$ propose that distinct sleep stages and the features of sleep preferentially support consolidation for different types of memory. Non-Rapid Eye Movement (NREM) sleep is involved in declarative memory consolidation, where sleep spindles (bursts
Correspondence: Stuart Fogel School of Psychology, University of Ottawa, Ottawa, Canada Email sfogel@uottawa.ca 
of $11-16 \mathrm{~Hz}$ oscillatory activity within thalamocortical networks) serve as an important marker of consolidation (for a review, $\mathrm{see}^{5}$ ). On the other hand, evidence from rapid eye movement (REM) sleep deprivation studies, ${ }^{6-8}$ REM-inhibition studies, ${ }^{9}$ targeted memory reactivation studies, ${ }^{10}$ and sleep recording studies ${ }^{11,12}$ suggest that REM sleep supports procedural memory consolidation, for skills that involve the acquisition of rules or novel strategies, whereby rapid eye movements serve as a marker of the consolidation process. ${ }^{13,14}$

The relationship between rapid eye movements and memory reprocessing is not fully understood. One yet unexplored explanation may be revealed by investigating the underlying neuronal activity that occurs concurrently with rapid eye movements. A potential marker of this learning effect may be theta activity. A wealth of literature has shown that hippocampal theta activity is involved in processes that support the encoding of memories, and in particular, long-term potentiation (LTP). ${ }^{15}$ LTP can be induced by stimulation patterns that mimic the theta rhythm ${ }^{16}$ and the strength of LTP increases linearly with theta power. ${ }^{17}$ Theta activity has also been associated with learning, for example, drugs that decrease theta power block learning ${ }^{18}$ while drugs that promote the theta rhythm facilitate learning. ${ }^{19,20}$ The hippocampus shows extensive projections (and back-projections) to the cortex, ${ }^{21}$ through which it is thought to induce theta activity. ${ }^{22}$ Cortical theta has been associated with sleep-related memory consolidation in rats $^{23}$ and humans. ${ }^{14}$ Hippocampal theta activity predominates REM sleep, ${ }^{24}$ and is correlated with rapid eye movements and ponto-geniculo-occipital (PGO) waves. $^{25-27}$ Taken together, there is sufficient evidence to suggest that theta activity is involved in sleep-dependent memory consolidation and that this is a likely candidate rhythm that may explain how rapid eye movements in REM sleep are markers of memory consolidation.

One theoretical framework for understanding how memory is involved in the acquisition of a second language is Ullman's Declarative-Procedural Model of SLL. ${ }^{1}$ Their model suggests that in the early stage of adult SLL, the declarative memory system is responsible for memorizing new word meanings, sounds and learning rudimentary grammar. However, as the SLL proceeds and experience in the second language accumulates, the acquisition of grammar shifts to depend increasingly on the procedural memory system (Figure 1). In support of this model, individual differences in declarative memory abilities are associated with early grammar skills, whereas individual differences in procedural memory abilities are associated with late grammar skills. ${ }^{28}$ Furthermore, procedural memory ability moderates grammatical performance for experienced adult second-language learning. ${ }^{29}$ Thus, from the perspective of Ullman's Declarative-Procedural model of SLL, we propose that memory functions during

\section{Ullman's Declarative-Procedural Model of SLL}

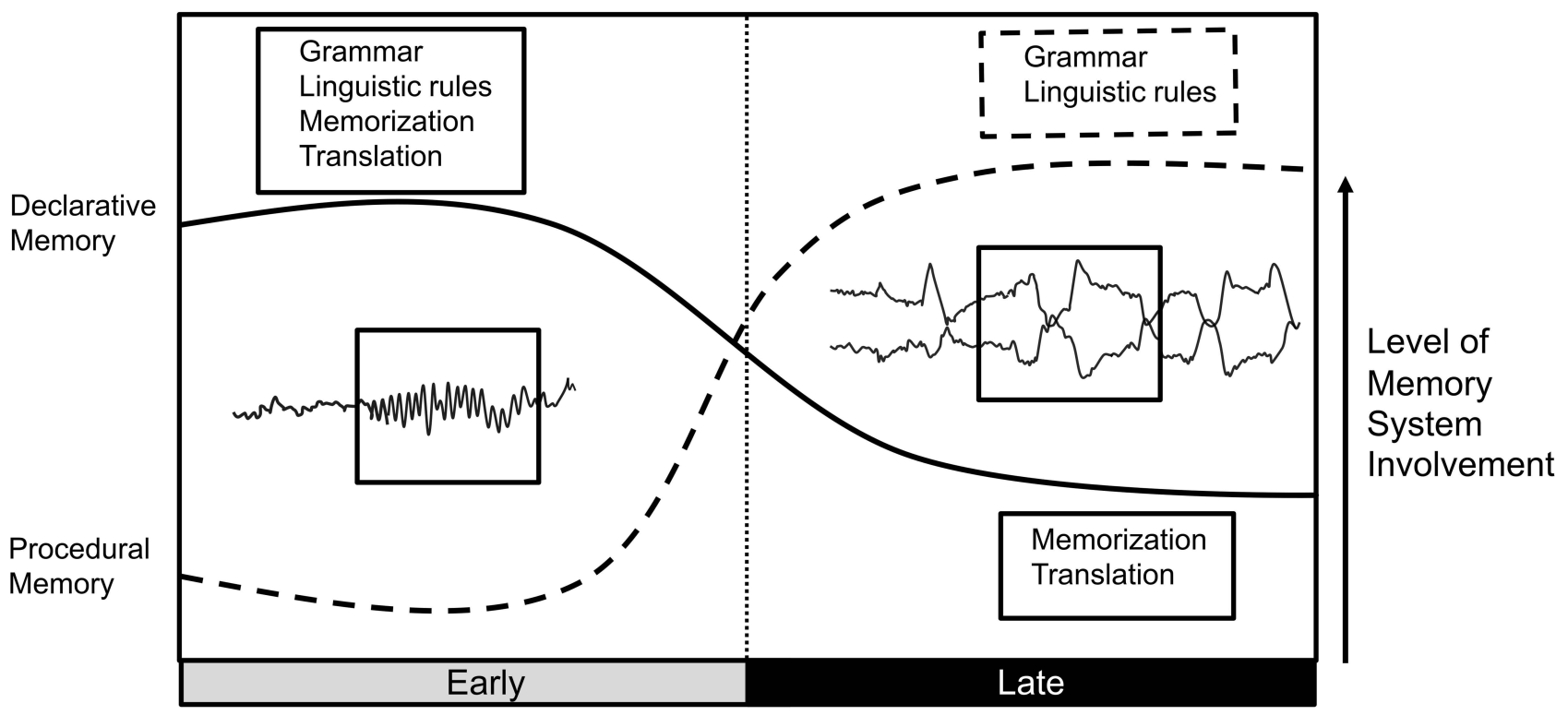

Stage of Second Language Acquisition

Figure I Representation of Ullman's Declarative-Procedural Model of SLL with an added role for electrophysiological sleep-associated markers of memory consolidation. 
NREM sleep (and sleep spindles) may be important for early language learning, while REM sleep (and rapid eye movements) may be important for late language learning.

Sleep supports acquiring a new language through offline memory processing. For example, a handful of previous studies and preliminary data have demonstrated that sleep enhances SLL in naturalistic settings, as a whole, ${ }^{30-35}$ as well as specific aspects of language learning including assigning meaning to words $^{36}$ enhancing phonological speech production, ${ }^{37}$ speech perception, ${ }^{38}$ integrating words into the mental lexicon, ${ }^{39}$ and learning implicit grammar. ${ }^{40}$ However, the idea that distinct sleep stages support the early versus late stages of second-language acquisition has yet to be directly investigated, nor is it known what features of sleep might support these distinct language stages.

NREM sleep has been found to support basic features of language learning such as speech perception. ${ }^{38}$ For example, sleep promotes abstraction of auditory probabilistic sequential structures, and the level of improvement in statistical learning (segment sequential speech into discrete words) is associated specifically with the amount of slow wave sleep (SWS) obtained. ${ }^{38}$ Furthermore, NREM sleep is implicated in aspects of language learning that contain a declarative component, such as assigning meaning to words ${ }^{36}$ and integrating words into the mental lexicon. ${ }^{39}$ Targeted memory reactivation using cues during NREM sleep improves vocabulary learning of foreign words with slow-waves and theta oscillations implicated as markers of this strengthening of memories during sleep. ${ }^{41}$ In children, sleep has been shown to consolidate the meaning of novel words, ${ }^{42}$ and spindle duration predicted task improvement in response to these words. $^{42}$ Together, this suggests that sleep (particularly sleep spindles and slow wave activity) may stabilize semantic linguistic information and aid in the integration of semantic linguistic information into memory networks.

By contrast, REM sleep is important for procedural memory consolidation of rules and strategies: abilities that are important for language processes such as grammatical skills. $^{40}$ Specifically, individuals with greater amounts of SWS and REM sleep during a post-learning nap show a greater sensitivity to learning a hidden linguistic rule. ${ }^{40}$ In a study that looked at language acquisition during an intensive second-language learning course, increases in REM sleep percentage were related to language learning efficiency. ${ }^{31}$ This increase corresponded to an enhancement of REM sleep during the second language course that then subsided after the course was completed. ${ }^{31}$ Taken together, NREM and REM sleep appear to be involved in SLL; however, it remains to be investigated if sleep spindles and rapid eye movements are electrophysiological markers of consolidation for SLL.

Approximately 30 years ago, a series of pioneering papers and proceedings investigated the role of sleep and dreams in a real-world SLL naturalistic paradigm. ${ }^{30-35}$ These studies employed the latest technology of the day: a 16 channel Model 4300 Nihon Kohden polygraph, which recorded EEG, EMG and EOG on paper and was capable of converting the analog signals to digital recordings that could be stored on a personal computer. Data from the present study originated from a subset of these seminal studies conducted in the summer of 1990-1991 at the University of Ottawa. It was the subject of three conference presentations $^{33-35}$ and was also the subject of a doctoral thesis. ${ }^{43}$ These studies amassed data in a total of $\mathrm{N}=18$ young adults with as many as 9-13 overnight recordings per individual (ie, almost 200 nights). At the time, very little was known about the functions of sleep. However, a series of groundbreaking animal studies by Smith et $\mathrm{al}^{44,45}$, Hennevin et $\mathrm{al}^{46}$, and Fishbein et $\mathrm{al}^{47}$ identified REM sleep as crucial for memory consolidation; periods of greatly increased REM sleep, termed REM sleep windows. Only a handful of studies up to this point were in humans, and several were theoretical works. $^{46-51}$ Notably, work by Smith et $\mathrm{al}^{8,10,52,53}$ and De Koninck $^{31-34}$ provided experimental evidence for a role of REM sleep in memory processing in humans. Here, this rare and valuable dataset was re-analyzed using novel methodology in order to address new unanswered questions about the electrophysiological correlates of SLA during sleep, using techniques that were not available at the time of the original study. Significant advancements in computing power, digital EEG processing, and analysis have made detecting and characterizing sleep features involved in memory processing widely available. In addition, these studies predate significant advancements in our understanding of the role of sleep in memory consolidation, as well as the role of declarative and procedural memory systems in SLL. As a result, several questions remain unanswered about how sleep supports SLL. For these reasons, we revisited data from these early studies, employing modern sleep and EEG analysis techniques. To explore this topic further, we have utilized the latest and novel methodologies to explore how the unique electrophysiological correlates (ie, rapid eye movements, sleep spindles) of sleep-associated memory consolidation support SLL (ie, early stage vs late stage). Here, we examined the relationship between neural oscillations during sleep (eg, spindles, theta) and improvements in French proficiency 
over the course of a 6-week intensive French second language program. We hypothesized that 1) when SLL is largely dependent on declarative memory, we would observe increased spindle-related activity (spindle density and size) during the early stage of SLL. It was expected that increases in spindles would be correlated with improvements in French proficiency; 2) when SLL is largely dependent on procedural memory, we would observe increased REM activity (eg, rapid eye movement density and EEG activity in the theta band time-locked to rapid eye movements) during the late stage of SLL. It was expected that increases in rapid eye movements and theta would be associated with improvements in French proficiency. Together, this research will advance our understanding of the role of sleep-associated markers of memory consolidation in supporting the acquisition of a second language in a naturalistic, real-world setting.

\section{Materials and Methods}

\section{Ethics Statement}

All participants provided written informed consent prior to the study and were financially compensated for their participation. The necessary research ethics approval was obtained within the School of Psychology at the University of Ottawa in accordance with the Declaration of Helsinki.

\section{Participants}

Thirteen participants were recruited for the study protocol from a 6 week-long intensive French as a second language course at the University of Ottawa's Second Language Institute. However, data from four participants could not be recovered from their storage media archive, and one participant was excluded for failing to comply with experimental protocols. A final sample of eight participants (5 female, $18-28$ years, $M_{\text {age }}=24$ years) were included in the current study. Participants were unilingual Anglophones that were enrolled in a post-secondary institution in southern Ontario, but outside of Ottawa(which is a bilingual city), in order to maximize the amount of language that they would need to learn. In fact, all participants completed a telephone screening interview to confirm they had minimal prior French language knowledge. They also had no signs of sleep disorders (eg, insomnia), psychological disorders (eg, severe anxiety, depression, phobias), medical conditions (eg, asthma, epilepsy), or neurological disorders (eg, head injury, brain damage, tumors). After the initial telephone screening, all potential participants visited the lab for a sleep acclimatization night and to screen for signs of sleep disorders, including apnea, movement disorders, and other parasomnias, using polysomnography (PSG; Figure 2 for the experimental protocol).

Participants without signs of sleep disorders completed a series of screening questionnaires including the Minnesota Multiphasic Personality Inventory (MMPI) to screen for signs of psychological or psychiatric conditions, the Edinburgh Handedness Inventory to identify handedness, the State-Trait Anxiety Inventory (STAI) to screen for situational and generalized anxiety, the Sixteen Personality Factor Questionnaire (16-PF) to screen for signs of psychological or psychiatric conditions and the Beck Depression Inventory (BDI) to screen for depression. All participants were required to abstain from daytime napping and from consuming alcohol on the days of polysomnographic recording. Participants included in the study were not taking any medications that might have impacted their sleep. To confirm compliance with experimental protocols, participants logged information about their daily activities, including notable events of their day, bedtime, wake time, medications taken, and any potential deviation from experimental guidelines including alcohol consumption throughout the duration of the study. Of note, throughout the course participants also maintained a dream diary, which is the subject of a separate study.

\section{Procedure}

Participants that met the inclusion and exclusion criteria were invited to come to Ottawa completed a series nine full polysomnographic sleep recordings at the University of Ottawa Sleep Research Laboratory throughout an intensive 6-week second language course (Figure 2): 1) the first night of the pre-course sleep recordings served to screen for sleep disorders, and the next two nights were for acclimatization purposes; 2) a series of two nights, the first to re-acclimatize to the sleep laboratory, and the second used for the early SLL night (during the course); 3) a second series of two nights, the first to re-acclimatize to the sleep laboratory, and the second used for the late SLL night (during the course); and 4) a final series of two nights, the first to re-acclimatize to the sleep laboratory, and the second used for the follow-up night (after the course), when learning-related changes in sleep were previously observed to have normalized. ${ }^{33-35}$ The sleep recordings consisted of electroencephalogram (EEG), electrooculogram (EOG) from the left and right outer canthus of the eye, chin electromyogram (EMG), and additional measures for nasal airflow and anterior lower leg muscles (see Polysomnographic Recording and Analysis, below for details). Participants completed the screening questionnaires 


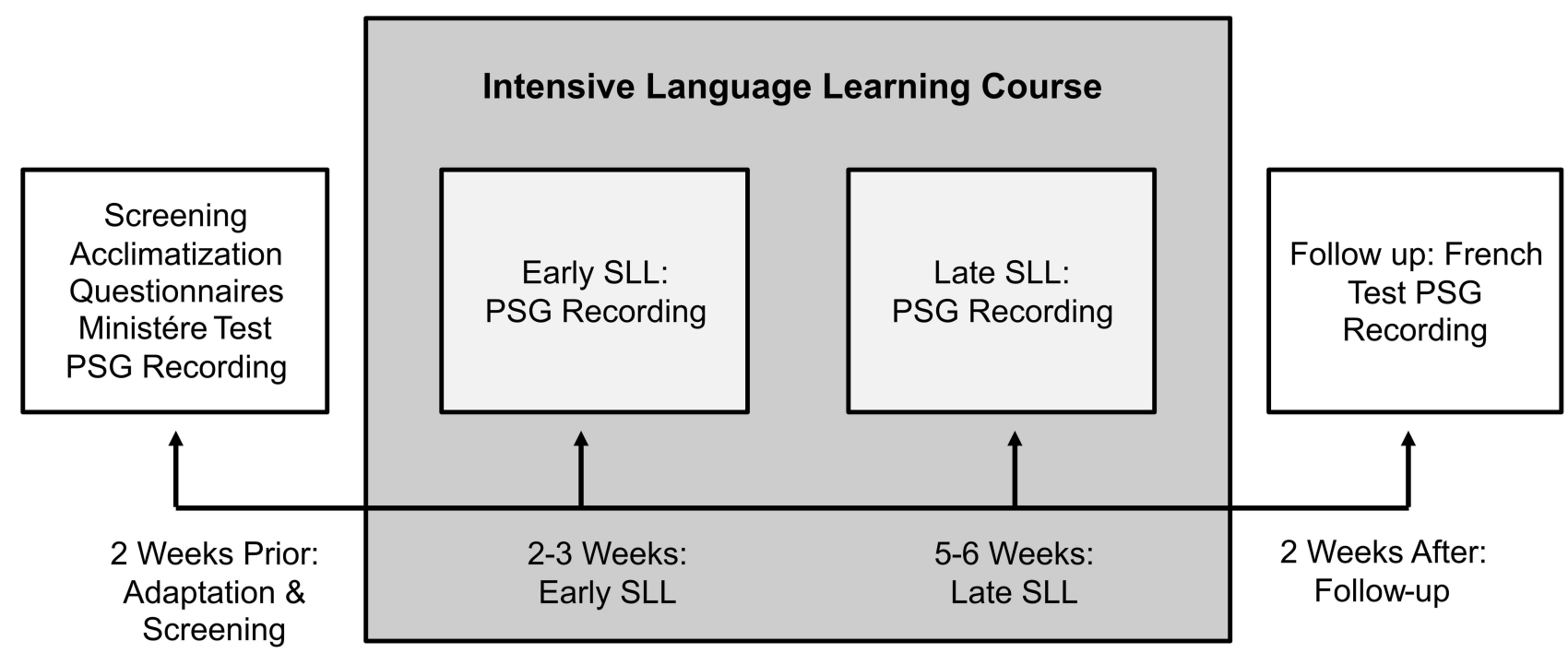

Figure 2 Experimental protocol. Participants first completed the "Screening and Acclimatization" nights (nights I-3) 2 weeks prior to beginning the course. During these nights, participants completed a battery of screening questionnaires, the Ministry French Proficiency Examination and three PSG recordings. Two to three weeks into the course, participants came back into the lab and completed the early acquisition PSG recordings (I: re-acclimatization, 2: early SLL night). Five to six weeks into the course, participants came back into the lab and completed the late acquisition PSG recordings (I: re-acclimatization, 2: late SLL night). Finally, 2 weeks after the course had ended, participants came back into the lab for the follow-up PSG recordings (I: re-acclimatization, 2: follow-up night).

and the Ministry French Proficiency Examination to assess their French language proficiency prior to, and at the end of the intensive second language course.

The early SLL night took place during either the second or third week of the intensive second-language learning course, the late SLL night took place during either the final fifth or sixth weeks of the course, and the follow-up night took place two weeks after the course concluded. Participants completed the Ministry French Proficiency Examination again on the evening of the follow-up night to assess their French language proficiency, following completion of the course. Note that the pre-course nights (screening and acclimatization), before the course started, were not used for comparison to early and late language learning nights as many of the participants experienced many other changes in their daily routines at that time, eg, living arrangements (moving to a new city) and lifestyle, prior to the outset of the course, which could have impacted their sleep patterns. Thus, the follow-up night ( 2 weeks after the course ended) was used to compare the impact of SLL on sleep during the course (early and late SLL) to sleep outside of the course, when there was adequate time for sleep patterns to have stabilized.

\section{Intensive Second Language Course}

The intensive second language course involved both formal and informal modes of learning. Formal French instruction was conducted through a program that ran from Monday to Friday (with occasional weekends) and included 6 hours of French language curriculum per day. Immersion was encouraged through 1) individually pairing students to share a room with a student whose first language was French, 2) organized social activities (eg, social gatherings, leisure activities, etc.) which gave students opportunities to practice French in social settings on evenings and weekends, and 3) an informal seminar series for the students was conducted throughout the course, which focused on language learning within the French-Canadian cultural context. Assuming complete immersion in the second language, this experience afforded up to about 672 hours of French language exposure and practice (16 hours per day, 7 days per week for 6 weeks).

\section{French Proficiency}

The French proficiency of the participants was assessed via the Ministry French Proficiency Examination; a measure consisting of 45 questions designed to assess oral, reading and writing comprehension administered by the Ministry of Education of the Government of Québec. Of the 45 questions, 25 questions measured the ability to understand spoken French. Twelve questions measured the ability to read and comprehend written French. Lastly, eight questions measured the ability to write in French. Scoring of the assessments was completed by the Ministry of Education of the Government of Québec. Higher scores indicate a higher proficiency in the French language. 


\section{Polysomnographic Recording and Analysis}

A Nihon Kohden Polygraph (Model 4314-B) EEG system was used to perform in-laboratory PSG recordings. Electrical activity recorded from EEG, EOG, and EMG channels were amplified by the polygraph, digitized by an A/D signal converter (Vetter Digital, model 400090) and stored on a microcomputer ( $\mathrm{PC} 486$ ) at a sampling rate of $256 \mathrm{~Hz}$. The data were filtered offline below $64 \mathrm{~Hz}$ with a low-pass filter. The data were then downsampled to 128 $\mathrm{Hz}$ and stored in European Data Format (EDF) on digital Write Once Read Many (WORM) optical drives. EEG was measured from two electrodes placed bilaterally at parietaltemporal sites (the W1 electrode was placed at the center of a triangle formed by the international 10-20 locations P3, T3, and T5; the W2 electrode was placed at the center of a triangle formed by the international 10-20 locations P4, T4, and T6). EEG and EOG, placed on the outer canthus of the left (LOC) and right (ROC) eyes, according to established standards and procedures were referenced to the mastoid electrodes (M1 and M2), which were linked in parallel to a $100-\mathrm{kohm}$ resistor. A submental EMG channel was recorded as a bipolar derivation. Manual sleep stage scoring according to standard criteria was completed by an expert polysomnographic technologist using SleepSMG (http:// sleepsmg.sourceforge.net/index.html), an EEGLAB compatible toolbox for MATLAB R2019a (MathWorks, Natick, Massachusetts, USA).

\section{EEG Analyses}

\section{EEG Preprocessing}

EEG data were digitally bandpass filtered in the MATLAB 2019a (MathWorks, Natick, Massachusetts, USA) toolbox EEGLAB; function "pop_eegfiltnew.m") on EEG channels (passband edges at $0.3-35 \mathrm{~Hz}$ ), EOG channels (passband edges at $0.3-10 \mathrm{~Hz}$ ), and the EMG channel (passband edges at $10-50 \mathrm{~Hz}$ ). Using custom MATLAB scripts, movement artifacts were identified on the EMG channel using a threshold approach with a cutoff of $25 \mu \mathrm{V}$. Automatically detected movement artifact events were then visually validated, and poor-quality EMG signals were manually scored, if necessary.

\section{Sleep Spindle Detection}

Sleep spindle events were identified at W1 and W2 electrodes from movement artifact-free, NREM2 sleep epochs in the EEG data. Automatic spindle detection was done using a previously validated method ${ }^{54}$ implemented in software compatible with EEGLAB. Briefly, this detection method extracts spindle-related phasic changes $(12-16 \mathrm{~Hz})$ through an individualized complex demodulation transformation of the EEG signal (see Ray et $\mathrm{al}^{54}$ for a detailed description of the method). The spindle variables extracted from the automated detection method were spindle size (duration $\times$ amplitude) and density (number of spindles per minute) for NREM2 sleep.

\section{Rapid Eye Movement Detection}

Eye movements during movement artifact-free REM sleep were detected using an adapted version of Yetton et $\mathrm{al}^{55}$ implementation of the Hatzilabrou et $\mathrm{al}^{56}$ matched filtering algorithm. This algorithm was chosen because, in comparison with other previously published algorithms, it was found to be the single best performer. ${ }^{55}$ As part of the matched filtering algorithm pipeline, ${ }^{56}$ the data were refiltered at $0.5-10 \mathrm{~Hz}$. After smoothing using a Hamming window, the correlation between a template of a typical eye movement and the LOC or ROC channel recordings was calculated to identify areas in the recording that matched the eye movement templates for eye movements. Monocular eye movements $<23 \mu \mathrm{V}$ were removed before the matched filtered output from ROC was inverted and subsequently multiplied by the output from the LOC channel. Eye movement events were marked by the detector at the maximum amplitude of the eye movement.

\section{Rapid Eye Movement Event-Related Spectral Perturbation}

To identify power spectral changes in the EEG signal that were time-locked to rapid eye movement events during REM sleep, an Event-Related Spectral Perturbation (ERSP) analysis was conducted. Using EEGLAB functions, each participant's data were segmented into 4-second epochs ( -2000 to $2000 \mathrm{~ms}$ ) centered on the peak of rapid eye movement. All participants had at least 700 eye movements identified and were included in the analysis. The EEGLAB function "newtimef.m" was used for the ERSP. The frequency range of interest was from 4 to 20 $\mathrm{Hz}$ to capture the theta $(4-8 \mathrm{~Hz})$, alpha $(8-12 \mathrm{~Hz})$, sigma (12$16 \mathrm{~Hz})$, and beta $(16-20 \mathrm{~Hz})$ frequency bands. Spectral power was baseline corrected using a divisive baseline from -1583 to $-460 \mathrm{~ms}$ in order to avoid overlap with the analysis window.

To determine the timing and duration of the average eye movement relative to the peak amplitude, the left and right EOG activity was time-locked to the eye movement peak marker and binned into separate categories, based on whether a positive or negative deflection occurred (Figure 3). The timing and duration of the average eye movement was used to determine the analysis window for 

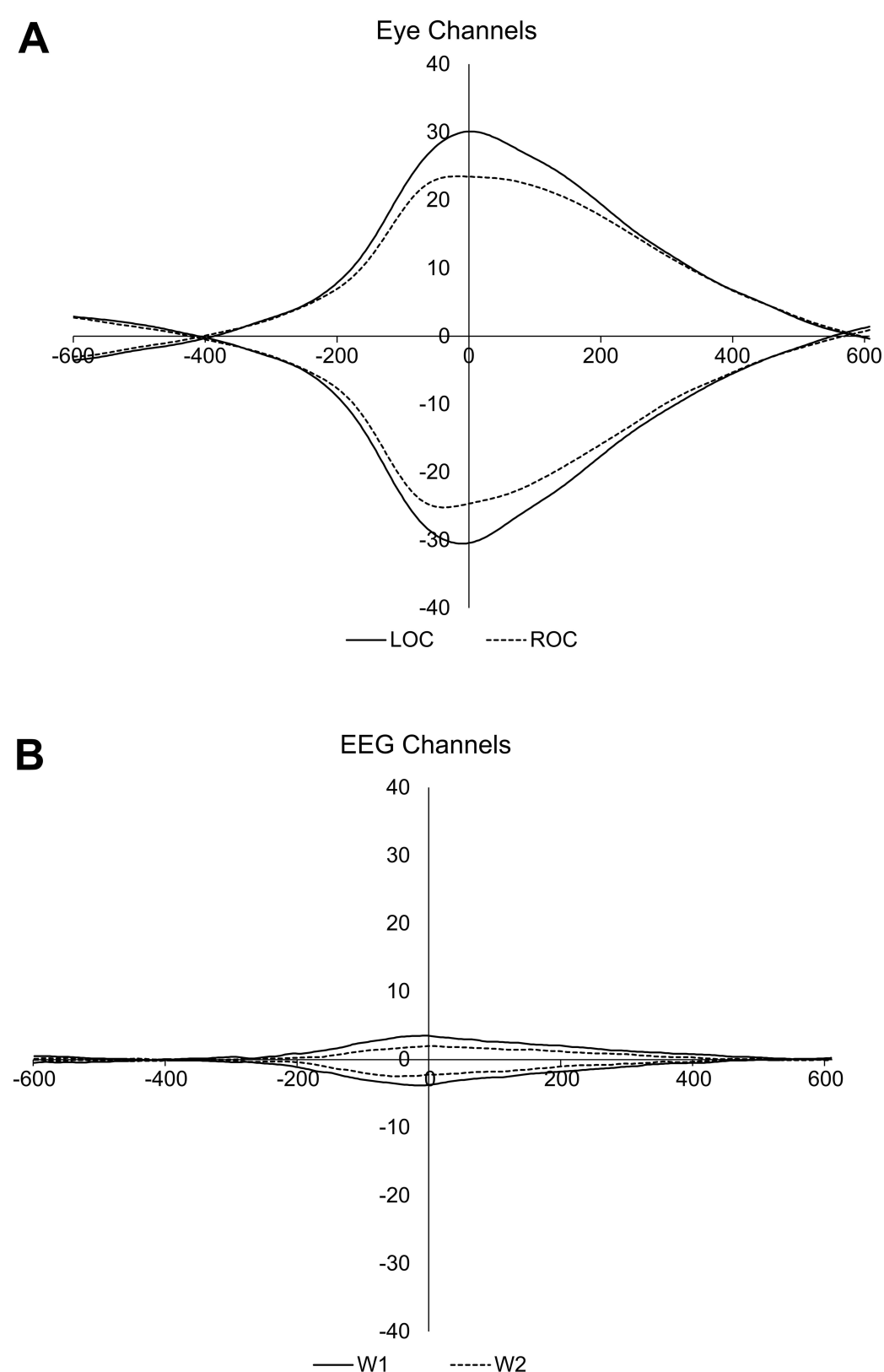

Figure 3 ERP plots of rapid eye movements during REM sleep. (A) Activity in EOG channels and for positive and negative deflections separately for LOC and ROC channels (B) activity in EEG WI and W2 channels time-locked to REM peaks in LOC and ROC channels. Note that eye movements did not induce significant movement artifacts on $\mathrm{WI}$ and W2; thus, changes in EEG spectral power are unlikely to be explained by artifact from night-to-night changes in eye movements.

subsequent analyses of the ERSP to -461 to $563 \mathrm{~ms}$. In addition, the event-related potentials aligned to the eye movement events for $\mathrm{W} 1$ and $\mathrm{W} 2$ were calculated to ensure that the ERSP analyses were not impacted by eyerelated artifacts (Figure 3B).

\section{Statistical Analysis}

Power Analysis and Effect Size Considerations

French Proficiency

A previous similar dataset ${ }^{31}$ using the same language learning course and French proficiency tests observed significant improvements in French proficiency from pre to post course based on a sample size of $n=10$ participants. Based on these behavioural effects, a power calculation was conducted for a within-subjects two-tailed $t$-test $($ alpha $=0.05)$ with an effect size of $d=1.48$, which indicated a total sample size of at least $n=6$ was sufficient for $80 \%$ power.

\section{Sleep Spindles}

A previous study $^{57}$ that examined learning-dependent 
changes in sleep spindle density, found a strong correlation $\left(r^{2}=0.76\right)$ between spindle density and learning improvements using $n=12$ participants. Based on this study, ${ }^{57}$ we conducted a power calculation for a two-tailed correlational analysis (alpha $=0.05,80 \%$ power) and determined a total sample size of at least $\mathrm{n}=5$ participants was sufficient.

\section{Rapid Eye Movements}

Relatively few studies investigated the relationship between rapid eye movements and memory. One of the most relevant studies to this investigation looked at REM density and complex procedural learning using a sample size of $\mathrm{n}=18^{13}$. Based on the study's effect size of $r^{2}=$ 0.31 , a power calculation (alpha $=0.05$ ) determined that we would need $n=16$ participants to achieve $80 \%$ power.

\section{REM Theta Power}

Previously, REM-related theta activity has been correlated with learning effects using $\mathrm{n}=9$ participants. ${ }^{14}$ This study was used to calculate power for a within-subjects twotailed $t$-test based on an effect size of $d=1.7$ and indicated a total sample size of $\mathrm{n}=5$ was sufficient for $80 \%$ power.

Based on these power analyses, we determined that our relatively small sample of $n=8$ participants would be sufficient to detect behavioural, sleep spindle, and REMrelated theta activity effects. However, we were likely underpowered to detect relationships between REM density and increases in French proficiency.

\section{French Proficiency}

To determine if French proficiency improved during the intensive language course, a paired-samples $t$-test was conducted on the Ministry French Proficiency Examination scores from before and after the course. For subsequent correlational analyses investigating the relationship between electrophysiological sleep events (eg, spindles, rapid eye movements) and improvements in French, improvement scores in French proficiency were computed as the difference between pre-course and post-course scores.

\section{Sleep Architecture}

One-way, repeated measures ANOVAs with SLL stage (early, late, follow-up) as the factor and sleep architecture variables as dependent measures were used to investigate how SLL impacted sleep architecture. The assumption of sphericity was not violated for any sleep architecture variables.

\section{Sleep Spindles}

To investigate the change in sleep spindle characteristics over the course of SLL, one-way repeated measures ANOVAs were conducted with stage of SLL as the factor (early, late, follow-up) and sleep spindle characteristics as the dependent variables (ie, density and size). The assumption of sphericity was not violated for the sleep spindle characteristics. Post-hoc paired samples $t$-tests were used to follow-up significant main effects from the ANOVA.

To further explore the relationship between night-tonight changes in sleep spindles and improvements in SLL, sleep spindle density and size across nights (early SLL to late SLL, early SLL to follow-up, late SLL to follow-up), for each electrode (W1 and W2), during NREM2 was entered into a correlational analysis with the French proficiency improvement scores. Kendall's Tau correlation coefficient was used as it is more robust for small sample sizes and is preferable to other non-parametric methods. ${ }^{58}$

\section{Rapid Eye Movements}

A one-way repeated measures ANOVA was used to investigate changes in rapid eye movement density across the stages of SLL (early, late, and follow-up). Kendall's Tau correlational analyses were also used to investigate the relationships between night-to-night changes in rapid eye movement density (early SLL to late SLL, early SLL to follow-up, and late SLL to follow-up) and improvements in French proficiency.

\section{Event-Related Spectral Perturbation (ERSP)}

Statistical analysis of the ERSP data was examined using a time range from -461 to $563 \mathrm{~ms}$, and a frequency range from $4 \mathrm{~Hz}$ to $20 \mathrm{~Hz}$. A cluster-based permutation test (alpha $=0.05, t$-statistic) on time-frequency data, using Fieldtrip functions ${ }^{59}$ implemented in EEGLAB was conducted to investigate the changes in power across nights (early SLL to late SLL, early SLL to follow-up, and late SLL to follow-up) for electrodes W1 and W2. A clusterbased analysis assumes that data points are not independent of one another and the values in neighbouring time or frequency points are likely to be related. A weighted cluster mean criterion was chosen, which considers both the cluster size and magnitude of the cluster test statistic. A Monte Carlo permutation statistical test was done with 10,000 randomizations.

To further explore the relationship between night-to-night changes in theta power time-locked to eye movements and improvements in SLL, significant changes in theta power 
Table I Mean (SEM) and ANOVA Results for Sleep Architecture on Early, Late, and Follow-Up Nights

\begin{tabular}{|c|c|c|c|c|c|c|}
\hline \multirow[t]{2}{*}{ Sleep Variable } & \multicolumn{3}{|c|}{ Night } & \multirow[b]{2}{*}{$\boldsymbol{F}$} & \multirow[b]{2}{*}{$p$} & \multirow[b]{2}{*}{$\eta_{p}{ }^{2}$} \\
\hline & Early & Late & Follow-Up & & & \\
\hline Total sleep time $(\mathrm{h})$ & $7.26(0.14)$ & $7.32(0.11)$ & $7.44(0.12)$ & 1.05 & 0.38 & 0.13 \\
\hline Sleep onset latency $(\mathrm{min})$ & $5.75(1.60)$ & $8.94(3.15)$ & $9.13(2.29)$ & 0.86 & 0.45 & 0.11 \\
\hline Sleep efficiency (\%) & $97.29(0.64)$ & $97.88(0.59)$ & $97.22(1.47)$ & 0.31 & 0.74 & 0.04 \\
\hline Wake after sleep onset (min) & $|2.3|(2.95)$ & $9.63(2.78)$ & $12.25(6.82)$ & 0.26 & 0.78 & 0.04 \\
\hline$\%$ NREMI & $1.36(0.29)$ & $1.32(0.42)$ & $\mathrm{I} .28(0.5 \mathrm{I})$ & 0.02 & 0.98 & 0.00 \\
\hline$\%$ NREM2 & $48.75(2.87)$ & $49.46(2.14)$ & $50.33(3.38)$ & 0.26 & 0.77 & 0.04 \\
\hline \% NREM3 & $24.82(3.13)$ & $22.72(2.28)$ & $23.40(3.58)$ & 1.01 & 0.39 & 0.13 \\
\hline$\%$ REM & $25.07(0.93)$ & $26.5 \mathrm{I}(2.05)$ & $24.99(1.83)$ & 0.34 & 0.72 & 0.05 \\
\hline
\end{tabular}

across nights (early SLL to late SLL) during REM sleep were entered into a correlational analysis (Kendall's Tau) with the French proficiency improvement scores.

\section{Results}

\section{Language Proficiency Results \\ Ministry French Proficiency Examination}

To investigate improvements in French proficiency following completion of the intensive second-language learning course, a paired $t$-test was used to compare differences in French proficiency from before the course to after the course concluded. The results revealed a significant increase in scores from pre- $(M=32.53, S E M=11.17)$ to post-course $(M=$ 56.53, SEM $=6.65, t(7)=4.75, \mathrm{p}=0.002, d=1.69)$, confirming that the students completing the intensive secondlanguage learning program significantly improved in French proficiency as a result of their participation in the program.

\section{Electrophysiological Results}

\section{Sleep Architecture}

Repeated measures ANOVAs were used to investigate changes in sleep architecture across stage of SLL (early
SLL, late SLL and Ffollow-up nights). There was no significant effect of stage of SLL for any sleep architecture variables (Table 1), indicating that significantly high level of REM $\%$ well above $20 \%$ and even $25 \%$ observed in a previous study (31) was attained in this case on average at the early period of the course so that sleep architecture did not change across the SLL.

\section{Sleep Spindles}

Repeated measures ANOVAs were used to investigate changes in sleep spindles across SLL stages (early SLL, late SLL and follow-up nights; Table 2). There was no effect of SLL stage on spindle density at either W1 ( $F$ $\left.(2,14)=0.16, p=0.86, \eta_{p}^{2}=0.02\right)$ or $\mathrm{W} 2$ electrodes $(F$ $\left.(2,14)=0.08, p=0.93, \eta_{p}^{2}=0.01\right)$. However, there was a significant effect of SLL stage on spindle size during $\operatorname{NREM} 2\left(F(2,14)=5.60, p=0.016, \eta_{p}{ }^{2}=0.44\right)$ on the left hemisphere, at electrode $\mathrm{W} 1$, but not on the right hemisphere at electrode $\mathrm{W} 2\left(F(2,14)=1.98, p=0.17, \eta_{p}{ }^{2}=\right.$ 0.2 ). Follow-up $t$-tests (Figure 4) revealed that this effect was driven by an increase in spindle size at early SLL compared with follow-up at $\mathrm{W} 1(t(7)=2.61, p=0.03, d=$ 0.86 ), as well as at late SLL compared with follow-up at

Table 2 Mean (SEM) and ANOVA Results for the Sleep Spindles (Size, Density) and REM Density Across the Nights (Early, Late, and Follow-Up)

\begin{tabular}{|c|c|c|c|c|c|c|c|c|}
\hline \multirow[t]{2}{*}{ Stage } & \multirow[t]{2}{*}{ Site } & \multirow[t]{2}{*}{ Variable } & \multicolumn{3}{|c|}{ Night } & \multirow[b]{2}{*}{$\boldsymbol{F}$} & \multirow[b]{2}{*}{$p$} & \multirow[b]{2}{*}{$\eta_{p}^{2}$} \\
\hline & & & Early & Late & Follow-Up & & & \\
\hline \multirow[t]{4}{*}{ NREM2 } & \multirow[t]{2}{*}{ WI } & Spindle Size & $16.43(1.62)$ & $16.92(1.3)$ & I3.64 (0.87) & 5.60 & 0.02 & 0.44 \\
\hline & & Spindle Density & $\mathrm{I} .6 \mathrm{I}(0.2 \mathrm{I})$ & $1.81(0.29)$ & $1.66(0.20)$ & 0.16 & 0.86 & 0.02 \\
\hline & \multirow[t]{2}{*}{ W2 } & Spindle Size & I5.90 (1.76) & I5.78 (0.63) & I3.89 (0.68) & 1.98 & 0.17 & 0.20 \\
\hline & & Spindle Density & I.64 (0.26) & $1.86(0.25)$ & I.7I (0.20) & 0.08 & 0.93 & 0.01 \\
\hline REM & & REM Density & $8.50(2.00)$ & $8.04(1.63)$ & 7.89 (2.02) & 0.11 & 0.77 & 0.02 \\
\hline
\end{tabular}




\section{W1 NREM2}

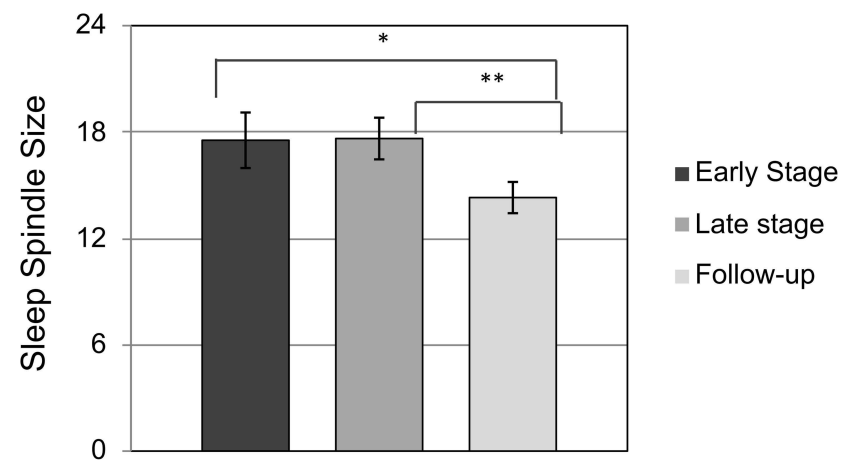

Figure 4 There was a significant increase in NREM2 spindle size at WI at the early stage of SLL compared with follow-up $(t(7)=2.6 \mathrm{I}, p=0.03, d=0.86)$, and a significant increase in spindle size at late SLL compared with follow-up $(t(7)=$ 4.35, $p=0.002, d=1.08$ ). Asterisks indicate significant results, ${ }^{*} p<0.05$, ** $p<$ 0.01 . Error bars denote standard error.

W1 $(t(7)=4.35, p=0.002, d=1.08)$. Thus, spindle size was larger during SLL than it was after the course concluded.

To investigate the relationship between improvements in French proficiency and sleep spindles, correlation coefficients were calculated between Ministry French Proficiency Examination differences scores and the change in spindle variables (density and size) from early SLL to late SLL, early SLL to follow-up, and from late SLL to follow-up (Table 3). There was a positive correlation between improvement in French proficiency and the change in spindle density from early SLL to late SLL during NREM2 $\left(r_{t}=0.64, p=0.03\right)$ at W1 (Figure 5). Together, this suggests that although there was not a significant change in spindle density over the course of SLL, individuals with a greater increase in spindle density between early and late SLL improved the most in French proficiency. Changes in spindle density for W2 from early to late $\left(r_{t}=0.07, p=0.80\right)$, early to follow-up $\left(r_{t}=-0.29\right.$, $p=0.32)$, or late to follow-up $\left(r_{t}=-0.36, p=0.22\right)$ were not significantly related to improvement in French proficiency. The change in spindle density at electrode $\mathrm{W} 1$ was not significantly related to improvements in French proficiency for early to follow-up $\left(r_{t}=0.21, p=0.46\right)$. Changes in spindle size were also not significantly correlated with improvements in French proficiency at either electrode W1 (early to late, $r_{t}=0.14, p=0.62$; early to follow-up, $r_{t}=$ $-0.14, p=0.62$; or late to follow-up, $r_{t}=-0.5, p=0.08$ ) or at electrode W2 (early to late, $r_{t}=-0.07, p=0.81$; early to follow-up, $r_{t}=-0.21, p=0.46$; or late to follow-up, $r_{t}=$ $-0.21, p=0.46$ ).

\section{Rapid Eye Movements}

A repeated measures ANOVA was used to investigate changes in REM density between the stages of SLL (early SLL, late SLL and follow-up nights). Counter to our initial hypotheses, there was no significant effect of night for REM density, suggesting that REM density did not differ between stages of second-language learning ( $F$ $(2,14)=0.11, p=0.77$; Table 2)

Kendall's Tau's correlational analyses were used to investigate the relationship between improvements in French proficiency and REM density. There was no significant correlation between improvements in French proficiency and changes in REM density for early to late $\left(r_{t}\right.$ $\left.=-0.29, p=0.32, \eta_{p}{ }^{2}=0.02\right)$, early to follow-up $\left(r_{t}=\right.$ $0.00, p=1)$, or late to follow-up $\left(r_{t}=0.29, p=0.32\right.$; Table 3). Thus, there was no association between REM density and SLL. However, the number of rapid eye movements may not be as informative about memory processes as the underlying neuronal activity that occurs concurrently, which was subsequently explored in the ERSP analyses.

Table 3 Kendall's Tau Correlation Results for the Change in Sleep Spindles (Density, Size) and REM Density Across the Nights (Early Late, Early - Follow-Up, Late - Follow-Up)

\begin{tabular}{|l|l|l|l|l|l|l|l|l|}
\hline \multirow{2}{*}{ Stage } & \multirow{2}{*}{ Site } & \multirow{2}{*}{ Variable } & \multicolumn{2}{l|}{ Early - Late } & \multicolumn{2}{l|}{ Early - Follow-Up } & \multicolumn{2}{l|}{ Late - Follow-Up } \\
\cline { 3 - 9 } & & & $\boldsymbol{r}_{\boldsymbol{t}}$ & $\boldsymbol{p}$ & $\boldsymbol{r}_{\boldsymbol{t}}$ & $\boldsymbol{p}$ & $\boldsymbol{r}_{\boldsymbol{t}}$ & $\boldsymbol{p}$ \\
\hline \multirow{3}{*}{ NREM2 } & \multirow{2}{*}{ WI } & Spindle Density & 0.64 & 0.03 & 0.21 & 0.46 & -0.50 & 0.08 \\
\cline { 3 - 10 } & & Spindle Size & 0.14 & 0.62 & -0.14 & 0.62 & -0.50 & 0.08 \\
\cline { 2 - 10 } & \multirow{2}{*}{ W2 } & Spindle Density & 0.07 & 0.80 & -0.29 & 0.32 & -0.36 & 0.22 \\
\cline { 3 - 10 } & & Spindle Size & -0.07 & 0.81 & -0.21 & 0.46 & -0.21 & 0.46 \\
\hline \multirow{2}{*}{ REM } & & REM Density & -0.29 & 0.32 & 0.00 & 1.00 & 0.29 & 0.32 \\
\hline
\end{tabular}




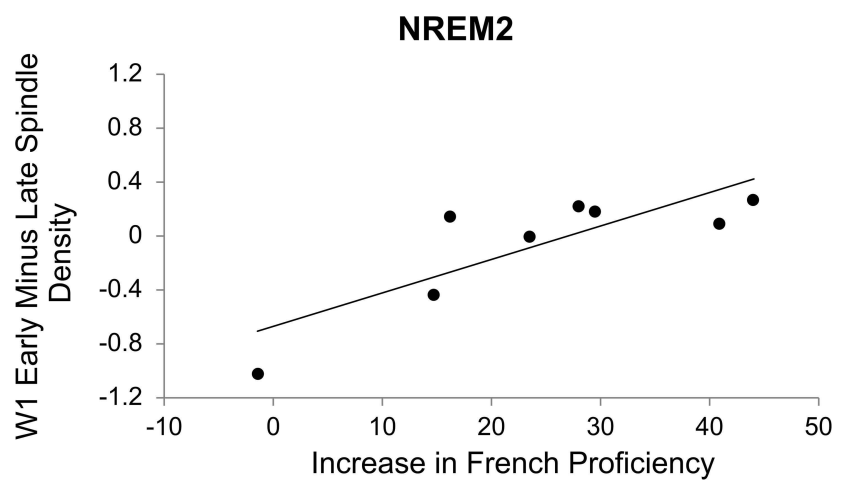

Figure 5 Scatterplots showing the relationship between the increase in French proficiency and early minus late spindle density at electrode WI for NREM2 ( $n=$ $0.64, p=0.03$ ).

\section{Event-Related Spectral Perturbation (ERSP)}

Power values from the ERSP were subjected to a nonparametric cluster-based permutation analysis to analyze the changes in event-related EEG power time-locked to rapid eye movements across nights (early vs late; early vs follow-up; and late vs follow-up) for each electrode (W1 and W2). There was a significant effect of stage of SLL at W1 that corresponded to increased power during the late stage of SLL compared with the early stage of SLL (cluster $t=3.67, p=0.03 ., d=1.17$; Figure 6). Increased power in the late stage of SLL compared with the early stage of SLL was observed approximately 100 $300 \mathrm{~ms}$ after the REM peak in the $4-8 \mathrm{~Hz}$ frequency range. No significant effects of change in power were found for early-vs.-follow-up or late-vs.-follow-up at W1, or between any of the conditions at W2 ( $\mathrm{p}>0.05)$.

Kendall's Tau's correlational analyses were used to investigate the relationship between improvements in French proficiency and increases in theta power from early to late SLL at electrode W1. This analysis revealed that there was a significant relationship $\left(r_{t}=0.63, p=\right.$ 0.026 ) between increases in theta power (from early to late stage SLL) and improvements in French proficiency (Figure 7).

\section{Discussion}

The present study revisited data from a previous series of pioneering studies ${ }^{30-35}$ with the aim of applying modern and novel analysis techniques in order to explore how sleep-associated markers of declarative and procedural long-term memory consolidation support the acquisition of a second language. Here, we show that 1a) spindle size was elevated during the intensive language course (both early and late SLL) compared with follow-up, 1b) improvements in French proficiency were significantly correlated with the change in spindle density from early SLL to late SLL (ie, participants who had more spindles during early SLL showed greater improvements in French proficiency), 2a) REM density was not elevated during the SLL course, and there was no association between changes in REM density and improvements in French proficiency, 2b) however, we show here for the first time an increase in theta activity time-locked to rapid eye movements at late SLL as compared to early SLL, and 2c) that the extent of improvements in French proficiency were significantly correlated with increases in theta power. These results extend on our previous knowledge about SLL and align well with Ullman's Declarative-Procedural model of SLL (Ullman, 2001) to include a role of sleep (see Figure 1). In sum, the present findings suggest that sleep spindles during NREM sleep support early (declarative-related) SLL, while theta activity time-locked to rapid eye movements during REM sleep support late (procedural-related) SLL.

We observed elevated spindle size while participants were completing the intensive second language course. This finding is consistent with research demonstrating that sleep spindles are involved in memory consolidation for both declarative and procedural memory. For example, changes in spindle characteristics have been identified in response to both declarative, ${ }^{60,61}$ cognitively complex procedural $^{62,63}$ memory consolidation as well as procedural skills memory. ${ }^{14,57,64-66}$ While this effect warrants further investigation, the present findings support the idea that sleep spindles may be involved in the consolidation of the expanding list of memory types they appear to support. Alternatively, while there is a shift for grammar from the declarative memory system to the procedural memory system with learning - given the naturalistic, yet intense nature of the course, it is very possible that new material was being acquired via declarative memory, even in the later stages of the course. Accordingly, engagement of the declarative memory system for SLL may have occurred throughout the course, which may explain the observed elevated spindle size at both early and late SLL.

In Ullman's Declarative-Procedural model of SLL, ${ }^{1}$ it is suggested that declarative memory is important for memorizing word meanings and sounds, as well as for acquiring and using grammar during the early phase of SLL. Sleep spindles are important for the optimal consolidation of declarative memory. For example, spindle number and density increase after learning a declarative 


\section{A}

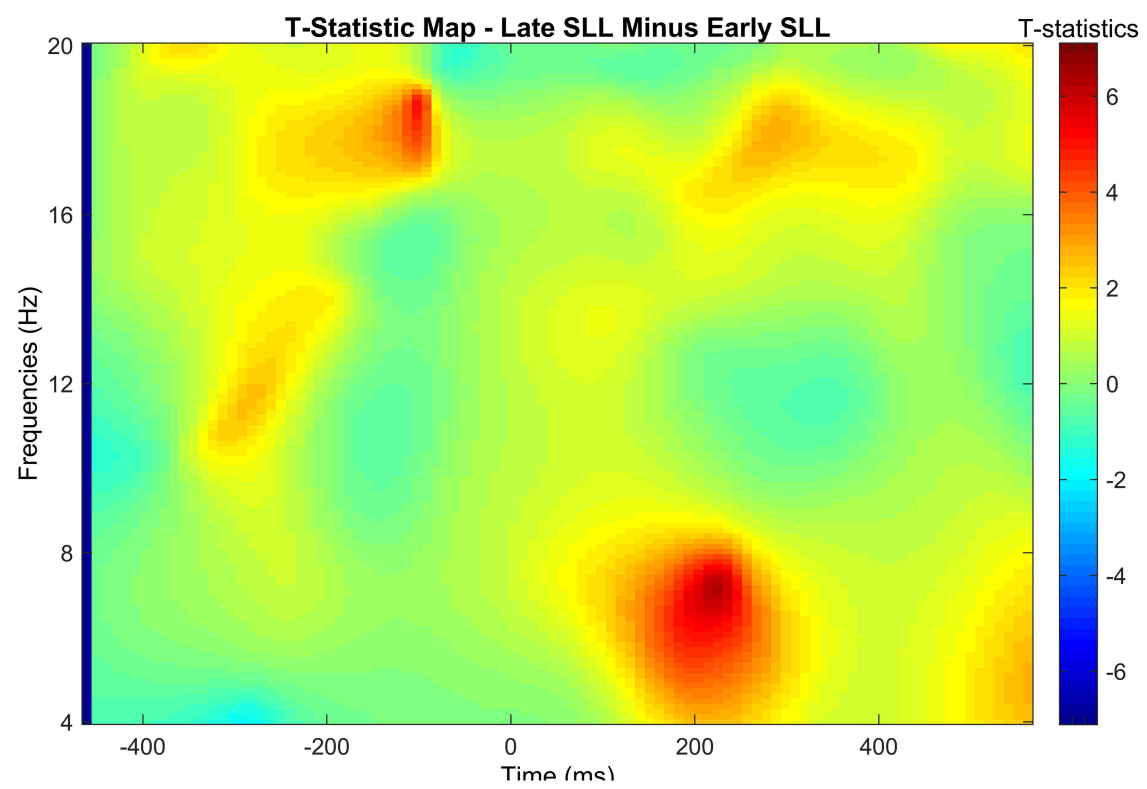

B

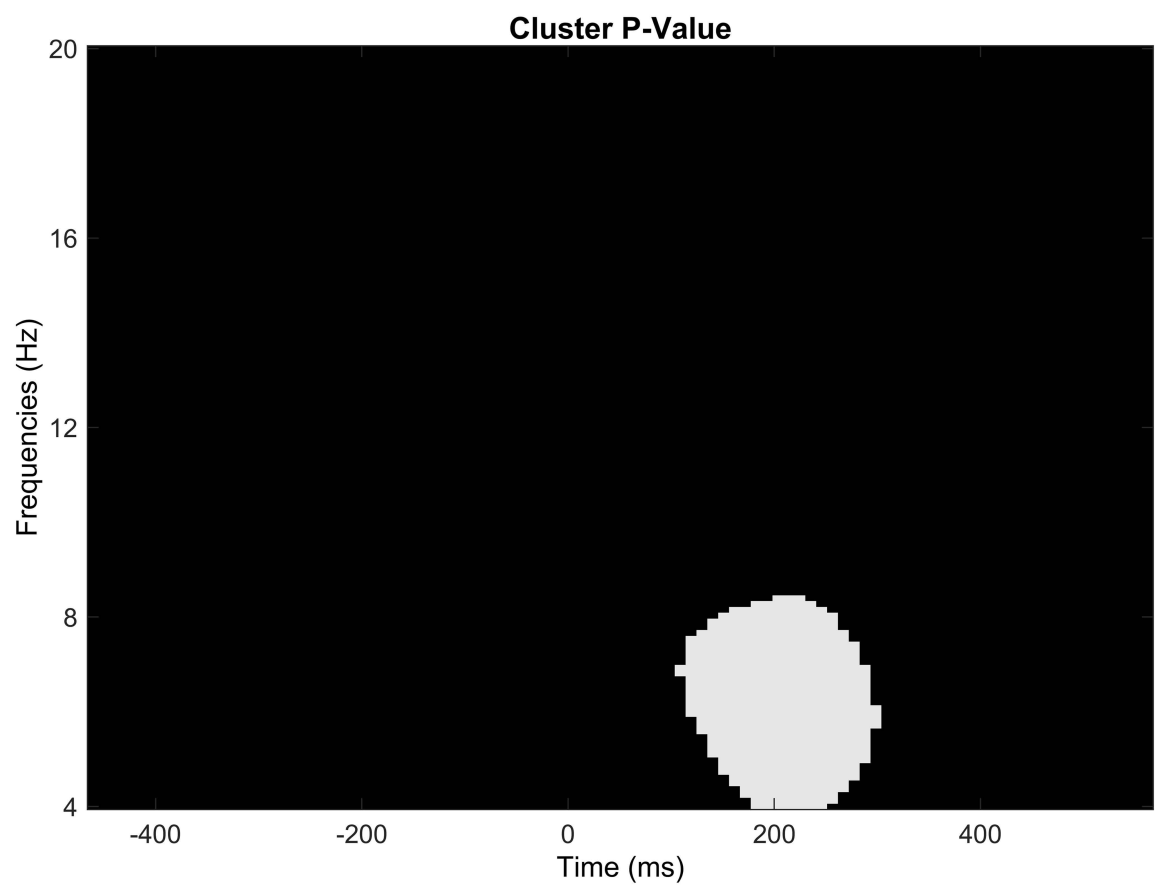

Figure 6 The $t$-map $(\mathbf{A})$ and statistical results for the cluster-based permutation analysis at WI (B), comparing late SLL versus early SLL $(p=0.03)$. Time 0 represents the peak of the rapid eye movement.

memory task and are correlated with task performance. $^{60,61,67,68}$ Enhancing spindles using transcranial alternating current enhances declarative memory performance. ${ }^{69}$ Moreover, spindle number, duration, and amplitude are reduced in neuropsychological cases of decreased declarative memory capacity. ${ }^{70}$ Here we show that there is an association between improvements in proficiency for a second language and increased spindle density from early to late stages of SLL. This finding is consistent with the function of sleep spindles for declarative memory consolidation and suggests that there is a specific period at the early phase of SLL (when declarative memory is most important), where sleep spindles may aid in consolidating the second language.

Rapid eye movements support memory consolidation for cognitively complex procedural skills. ${ }^{5}$ However, why eye movements per se are important markers of this process 


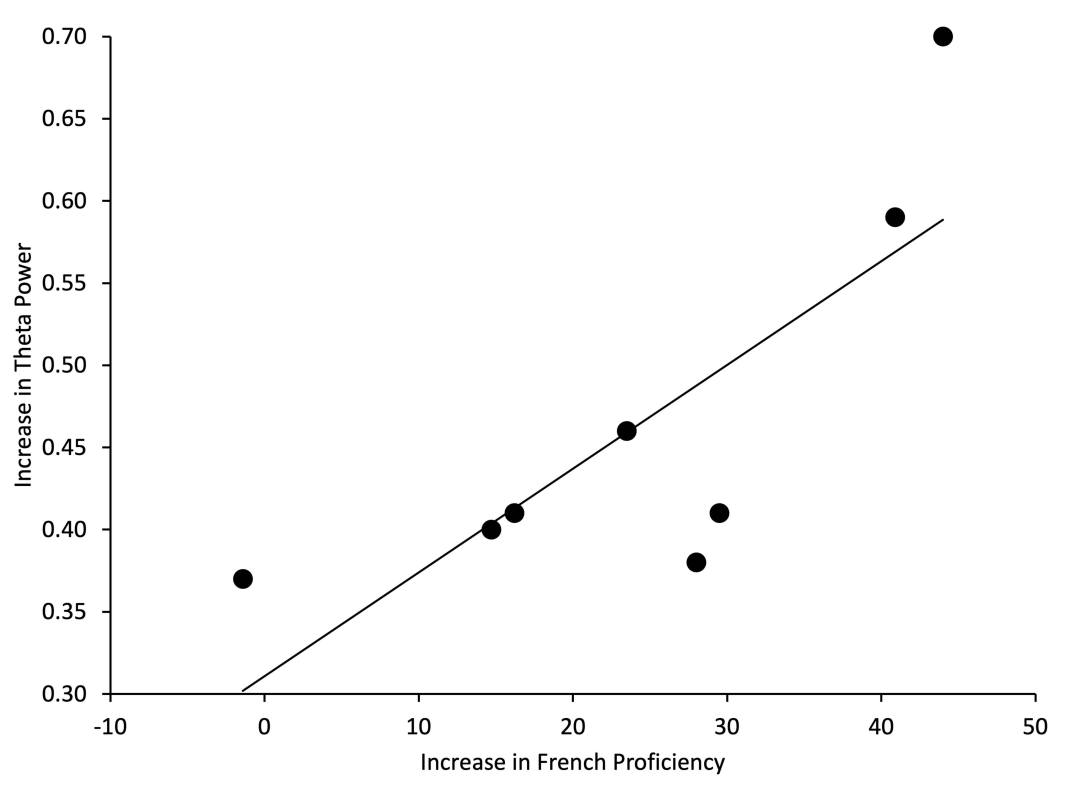

Figure 7 Scatterplot of the relationship between the increase in French proficiency and the change in theta power from early to late stage of SLL (late minus early) timelocked to eye movements during REM sleep $(n=0.63, p=0.026)$.

remains to be answered. In Ullman's Declarative-Procedural model of SLL, ${ }^{1}$ it is proposed that during the late phase of second-language learning there is a shift from declarative to procedural memory systems for the acquisition and use of grammar. Memory for cognitively complex procedural skills has been shown to be consolidated during REM sleep. ${ }^{6,8,10,13,14,71-73}$ The number and density of eye movements during REM are correlated with task performance improvements, ${ }^{13}$ and are considered a marker for the intensity of this memory function of REM sleep. Rapid eye movements are generated by rhythmic oscillations in the ponto-geniculooccipital circuit. Beyond that, the underlying associated neural activity that might link eye movements to memory processing remains a mystery. Contrary to our hypotheses, the current study did not identify any changes in REM density during late SLL, or any correlation between REM density and language proficiency. Instead, here, we show for the first time, an increase in EEG theta activity time-locked to rapid eye movements during a period of learning (ie, cognitively complex procedural learning) where memory consolidation is thought to be REM associated. Importantly, we observed that the significant enhancements in theta power were correlated with the extent of French language improvement. Thus, suggesting that REM-related changes in theta power were specifically related to SLL. Theta activity has been linked with LTP, ${ }^{16}$ learning processes, ${ }^{18-20}$ and sleep-related memory consolidation in rats. ${ }^{23}$ Hippocampal theta activity predominates REM sleep, ${ }^{24}$ and shows an association with both rapid eye movements and PGO waves. ${ }^{25-27}$ The hippocampus shows projections (and back-projections) to the cortex, ${ }^{21}$ through which it is thought to induce theta activity. ${ }^{22}$ Together, this may suggest there is an underlying memory process related to theta activity that is coincident with rapid eye movements during REM sleep. Whether this association is linked to the eye movements themselves or with PGO waves that generate eye movements is unclear and should be investigated in future studies. Rapid eye movements are a marker of the intensity of REM sleep; and it is well established that they are linked to PGO waves. ${ }^{74,75}$ However, it is not possible to study PGO activity in humans directly without the use of highly invasive recordings; thus, with few exceptions, the functional significance of rapid eye movements can only be studied indirectly. While rapid eye movements are mechanistically linked to PGO waves, this does not preclude the possibility that other brain activity might be associated with PGO activity or the related occurrence of rapid eye movements. Nor does the presence of spectral perturbations in the EEG time-locked to eye movements preclude the well-established role of the PGO circuitry in the generation of REMs. Additional research is required to investigate the link between PGO-related rapid eye movements and brain activity outside this well-known phenomenon.

It is interesting to note that participants in this study exhibited high percentages of REM sleep across all recordings at the beginning of the course to post course (averages of $25-26 \%$ ), which is consistent with elevated levels observed 
in previous studies involving intensive complex learning, eg, visual inversion and language learning. ${ }^{76}$ Indeed, even recent normative studies report typical REM sleep percentages in this age group at about $19 \%$ to $21 \%{ }^{77}$ The particularly high levels of REM sleep in this case may be due to the fact that all subjects experienced many other changes in their daily routines, living arrangements (eg, moving to a new city) and lifestyle at the onset of the study, in addition to the ongoing language learning. Thus, REM sleep duration may have been at, or approached some sort of ceiling for natural REM production. This observation makes it important to examine micro components such as those provided here to identify language learning-related changes in sleep in comparison to outside of the intensive language learning course.

The main limitation of the current study was the small final sample size $(\mathrm{N}=8)$, which limited our ability to detect small to medium sized effects. A post-hoc sensitivity power analysis in $G^{*}$ Power revealed that the current investigation was only able to detect large effect sizes, but importantly, was sufficiently powered to do so. Thus, the null results reported here should be taken with caution. However, despite the small sample size, we were still able to extend important previous findings and show robust effects, which warrant follow-up. In addition, the current study would be strengthened by comparing to a nonlanguage learning control group. Importantly, we did have a post-course follow-up overnight sleep recording, where sleep parameters were previously observed to have returned to baseline ${ }^{33-35}$ in an extensive and powerful repeated measures design. Nonetheless, for this reason, the results should be considered proof-of-concept, warranting replication in a larger sample as it cannot be conclusively determined if the observed results were due to some non-specific aspect of being enrolled in the course, or some other unknown factors occurring systematically over time.

\section{Conclusion}

In conclusion, the present study suggests that sleep spindles may contribute to declarative memory consolidation for aspects of SLL such as word meaning, sounds, and grammar during the early phase of SLL, whereas theta activity timelocked to the peak of the eye movements during REM sleep may be important during late SLL, and may relate to procedural learning, and in particular grammatical rules. This novel theta activity finding warrants further investigation in a larger sample, in a variety of REM-related memory tasks and paradigms. In summary, this study has implications for our understanding of how sleep-related memory consolidation is associated with the electrophysiological features of sleep, as well as expands our understanding of how second languages are acquired in a naturalistic setting. These findings may contribute to our understanding of language impairments, as problems in language learning are associated with sleep disorders for both children and adults. ${ }^{78}$ Furthermore, given the proposed involvement of declarative and procedural memory systems in first-language acquisition, ${ }^{1}$ the present research may be extended to our understanding of first-language acquisition as well. This study demonstrates for the first time employing a naturalistic paradigm that NREM sleep and spindles in particular support declarative aspects of early SLL, and that REM sleep and cortical theta activity time-locked to rapid eye movements support procedural aspects of late SLL.

\section{Acknowledgments}

This study was supported by a grant to JDK from the Natural Sciences and Engineering Research Council (NSERC) and the Social Sciences and Humanities Research Council of Canada (SSHRC), and an NSERC graduate scholarship to $\mathrm{GH}$.

\section{Disclosure}

The authors report no conflicts of interest in this work.

\section{References}

1. Ullman MT. A neurocognitive perspective on language: the declarative/procedural model. Nat Rev Neurosci. 2001;2(10):717-726. doi: $10.1038 / 35094573$

2. Peigneux P, Fogel SM, Smith CT. Memory processing in relation to sleep. In: Kryger HM, Roth T, Dement WC, editors. Principles and Practice of Sleep Medicine: Fifth Edition. 6th ed. Elsevier; 2015:335-347. doi:10.1016/B978-1-4160-6645-3.00029-3

3. Rauchs G, Desgranges B, Foret J, Eustache F. The relationships between memory systems and sleep stages. J Sleep Res. 2005;14 (2):123-140. doi:10.1111/j.1365-2869.2005.00450.x

4. Diekelmann S, Born J. The memory function of sleep. Nat Rev Neurosci. 2010;11(2):114-126. doi:10.1038/nrn2762

5. Fogel SM, Smith CT. The function of the sleep spindle: a physiological index of intelligence and a mechanism for sleep-dependent memory consolidation. Neurosci Biobehav Rev. 2011;35(5):1154-1165. doi:10.1016/j.neubiorev.2010.12.003

6. Plihal W, Born J. Effects of early and late nocturnal sleep on declarative and procedural memory. J Cogn Neurosci. 1997;9(4):534-547. doi:10.1162/jocn.1997.9.4.534

7. Plihal W, Born J. Memory consolidation in human sleep depends on inhibition of glucocorticoid release. Neuroreport. 1999;10 (13):2741-2747. doi:10.1097/00001756-199909090-00009

8. Smith CT. REM sleep and learning: some recent findings. In: Moffitt Kramer M, Hoffmann RA, editors. Funct Dreaming; 1993:341-361. Suny Press.

9. Smith CT, Smith D. Ingestion of ethanol just prior to sleep onset impairs memory for procedural but not declarative tasks. Sleep. 2003;26(2):185-191. doi:10.1093/sleep/26.2.185 
10. Smith CT, Weeden K. Post training REMs coincident auditory stimulation enhances memory in humans. Psychiatr J Univ Ott. 1990;15 (2):85-90.

11. Buchegger J, Fritsch R, Meier-Koll A, Riehle H. Does trampolining and anaerobic physical fitness affect sleep? Percept Mot Skills. 1991;73(1):243-252. doi:10.2466/pms.1991.73.1.243

12. Buchegger J, Meier-Koll A. Motor learning and ultradian sleep cycle: an electroencephalographic study of trampoliners. Percept Mot Skills. 1988;67(2):635-645. doi:10.2466/pms.1988.67.2.635

13. Smith CT, Nixon M, Nader RS. Posttraining increases in REM sleep intensity implicate REM sleep in memory processing and provide a biological marker of learning potential. Learn Mem. 2004;11 (6):714-719. doi:10.1101/1m.74904

14. Fogel SM, Smith CT, Cote KA. Dissociable learning-dependent changes in REM and non-REM sleep in declarative and procedural memory systems. Behav Brain Res. 2007;180(1):48-61. doi:10.1016/ j.bbr.2007.02.037

15. Vertes RP. Hippocampal theta rhythm: a tag for short-term memory. Hippocampus. 2005;15(7):923-935. doi:10.1002/hipo.20118

16. Larson J, Wong D, Lynch G. Patterned stimulation at the theta frequency is optimal for the induction of hippocampal long-term potentiation. Brain Res. 1986;368(2):347-350. doi:10.1016/00068993(86)90579-2

17. Maren S, DeCola JP, Swain RA, Fanselow MS, Thompson RF. Parallel augmentation of hippocampal long-term potentiation, theta rhythm, and contextual fear conditioning in water-deprived rats. Behav Neurosci. 1994;108(1):44-56. doi:10.1037/0735-7044.108.1.44

18. Givens B, Olton DS. Bidirectional modulation of scopolamine-induced working memory impairments by muscarinic activation of the medial septal area. Neurobiol Learn Mem. 1995;63(3):269-276. doi:10.1006/ nlme.1995.1031

19. Staubli U, Xu FB. Effects of 5-HT3 receptor antagonism on hippocampal theta rhythm, memory, and LTP induction in the freely moving rat. $J$ Neurosci. 1995;15(3II):2445-2452. doi:10.1523/jneurosci.15-0302445.1995

20. Staubli U, Rogers G, Lynch G. Facilitation of glutamate receptors enhances memory. Proc Natl Acad Sci U S A. 1994;91(2):777-781. doi:10.1073/pnas.91.2.777

21. Miller R. Cortico-Hippocampal Interplay and the Representation of Contexts in the Brain; 2013. Springer Science \& Business Media.

22. Klimesch W. Memory processes, brain oscillations and EEG synchronization. Int $J$ Psychophysiol. 1996;24(1-2):61-100. doi:10.1016/S0167-8760(96)00057-8

23. Fogel SM, Smith CT, Beninger RJ. Evidence for 2-stage models of sleep and memory: learning-dependent changes in spindles and theta in rats. Brain Res Bull. 2009;79(6):445-451. doi:10.1016/j. brainresbull.2009.03.002

24. Buzsaki G. Theta oscillations in the hippocampus. Neuron. 2002;33 (3):325-340. doi:10.1016/S0896-6273(02)00586-X

25. Karashima A, Katayama N, Nakao M. Phase-locking of spontaneous and tone-elicited pontine waves to hippocampal theta waves during REM sleep in rats. Brain Res. 2007;1182:73-81. doi:10.1016/j. brainres.2007.08.060

26. Karashima A, Nakao M, Honda K, Iwasaki N, Katayama N, Yamamoto $\mathrm{M}$. Theta wave amplitude and frequency are differentially correlated with pontine waves and rapid eye movements during REM sleep in rats. Neurosci Res. 2004;50(3):283-289. doi:10.1016/j. neures.2004.07.007

27. Karashima A, Nakao M, Katayama N, Honda K. Instantaneous acceleration and amplification of hippocampal theta wave coincident with phasic pontine activities during REM sleep. Brain Res. 2005;1051(12):50-56. doi:10.1016/j.brainres.2005.05.055

28. Morgan-Short K, Faretta-Stutenberg M, Brill-Schuetz KA, Carpenter H, Wong PCM. Declarative and procedural memory as individual differences in second language acquisition. Bilingualism. 2014;17(1):56-72. doi:10.1017/S1366728912000715
29. Granena G. Individual differences in sequence learning ability and second language acquisition in early childhood and adulthood. Lang Learn. 2013;63(4):665-703. doi:10.1111/lang.12018

30. De Koninck J, Christ G, Rinfret N, Proulx G. Dreams during language learning: when and how is the new language integrated. Psychiatr J Univ Ott. 1988;13(2):72-74.

31. De Koninck J, Lorrain D, Christ G, Proulx G, Coulombe D. Intensive language learning and increases in rapid eye movement sleep: evidence of a performance factor. Int J Psychophysiol. 1989;8(1):43-47. doi:10.1016/0167-8760(89)90018-4

32. De Koninck J, Christ G, Hébert G, et al. Language learning efficiency, dreams and REM sleep. Psychiatr J Univ Ott. 1990;15(2):91-92.

33. Hébert G, De Koninck J, Wecklein M. Hemispheric activity in REM sleep and language learning. Paper presented at the annual meeting of the association of professional sleep societies, phoenix, Arizona. Abstr Publ Sleep Res. 1992;21:104.

34. Hébert, G., \& De Koninck, J. EEG interhemispheric shift during REM sleep in second language learners. Paper presented at the Annual Meeting of the Association of Professional Sleep Societies, Los Angeles. Abstract published in Sleep Research. 1993;22:87.

35. Hébert G, De Koninck J. Frontal and parieto-temporal EEG in REM sleep during second language learning. J Sleep Res. 1994;3:100.

36. Tamminen J, Lambon Ralph MA, Lewis PA. The role of sleep spindles and slow-wave activity in integrating new information in semantic memory. $J$ Neurosci. 2013;33(39):15376-15381. doi:10.1523/JNEUROSCI.5093-12.2013

37. Gaskell MG, Warker J, Lindsay S, et al. Sleep underpins the plasticity of language production. Psychol Sci. 2014;25(7):1457-1465. doi:10.1177/0956797614535937

38. Durrant SJ, Taylor C, Cairney SA, Lewis PA. Sleep-dependent consolidation of statistical learning. Neuropsychologia. 2011;49 (5):1322-1331. doi:10.1016/j.neuropsychologia.2011.02.015

39. Tamminen J, Payne JD, Stickgold R, Wamsley EJ, Gaskell MG. Sleep spindle activity is associated with the integration of new memories and existing knowledge. $J$ Neurosci. 2010;30 (43):14356-14360. doi:10.1523/JNEUROSCI.3028-10.2010

40. Batterink LJ, Oudiette D, Reber PJ, Paller KA. Sleep facilitates learning a new linguistic rule. Neuropsychologia. 2014;65:169-179. doi:10.1016/j.neuropsychologia.2014.10.024

41. Schreiner T, Rasch B. Boosting vocabulary learning by verbal cueing during sleep. Cereb Cortex. 2015;25(11):11. doi:10.1093/cercor/bhu139

42. Fletcher FE, Knowland V, Walker S, Gaskell MG, Norbury C, Henderson LM. Atypicalities in sleep and semantic consolidation in autism. Dev Sci. 2019;23(3):e12906. doi:10.1111/desc.12906

43. Hébert G. L'activité interhémisphérique en sommeil paradoxal durant l'apprentissage intensif d'une langue seconde[Interhemispherical activity in REM sleep during intensive second language learning]. 1994. doi:10.20381/ruor-15069

44. Smith CT. Sleep states and memory processes. Behav Brain Res. 1995;69(1-2):137-145. doi:10.1016/0166-4328(95)00024-n

45. Smith CT. Sleep states and learning: a review of the animal literature. Neurosci Biobehav Rev. 1985;9(2):157-168. doi:10.1016/01497634(85)90042-9

46. Hennevin E, Leconte P. La fonction du sommeil paradoxal: faits et hypothèses. Lannee psychologique. 1971;71(2):489-519. doi:10.3406/ PSY.1971.27756

47. Fishbein, W., Kastaniotis, C., \& Chattman, D (1974). Paradoxical sleep: prolonged augmentation following learning. Brain research. 79(1):61-75.

48. Empson JAC, Clarke PR. Rapid eye movements and remembering. Nature. 1970;227(5255):287-288. doi:10.1038/227287a0

49. Dujardin K, Guerrien A, Mandai O, Sockeel P, Leconte P. Facilitation mnésique par stimulation auditive au cours du sommeil paradoxal (SP) chez l'homme. C R Acad Sci III. 1988;307(11):653-656.

50. Schoen LS, Badia P. Facilitated recall following RFM and NREM naps. Psychophysiology. 1984;21(3):299-306. doi:10.1111/J.14698986.1984.TB02937.X 
51. Greenberg R, Pearlman C, Schwartz WR, Grossman HY. Memory, emotion, and REM sleep. J Abnorm Psychol. 1983;92(3):378-381. doi:10.1037/0021-843X.92.3.378

52. Aubrey JB, Smith CT, Tweed S, Nader RS. Cognitive and motor procedural tasks are dissociated in REM and stage two sleep. Sleep Res Online. 1999;2(Suppl. 1):220.

53. Lapp L, Smith CT. The effect of one night's sleep loss on learning and memory in humans. Sleep Res. 1986;15:73.

54. Ray LB, Sockeel S, Soon M, et al. Expert and crowd-sourced validation of an individualized sleep spindle detection method employing complex demodulation and individualized normalization. Front Hum Neurosci. 2015;9(9). doi:10.3389/fnhum.2015.00507

55. Yetton BD, Niknazar M, Duggan KA, et al. Automatic detection of rapid eye movements (REMs): a machine learning approach. J Neurosci Methods. 2016;259:72-82. doi:10.1016/j.jneumeth.2015.11.015

56. Hatzilabrou GM, Greenberg N, Sclabassi RJ, Carroll T, Guthrie RD, Scher MS. A comparison of conventional and matched filtering techniques for rapid eye movement detection of the newborn. IEEE Trans Biomed Eng. 1994;41(10):990-995. doi:10.1109/10.324532

57. Fogel SM, Smith CT. Learning-dependent changes in sleep spindles and stage 2 sleep. J Sleep Res. 2006;15(3):250-255. doi:10.1111/ j.1365-2869.2006.00522.x

58. Croux C, Dehon C. Influence functions of the Spearman and Kendall correlation measures. Stat Methods Appt. 2010;19(4):497-515. doi:10.1007/s10260-010-0142-Z

59. Oostenveld R, Fries P, Maris E, Schoffelen J-M. FieldTrip: open source software for advanced analysis of MEG, EEG, and invasive electrophysiological data. Comput Intell Neurosci. 2011;2011:156869. doi:10.1155/2011/156869

60. Schabus M, Gruber G, Parapatics S, et al. Sleep spindles and their significance for declarative memory consolidation. Sleep. 2004;27 (8):1479-1485. doi:10.1093/sleep/27.7.1479

61. Schabus M, Hoedlmoser K, Pecherstorfer T, et al. Interindividual sleep spindle differences and their relation to learning-related enhancements. Brain Res. 2008;1191:127-135. doi:10.1016/j. brainres.2007.10.106

62. van Schalkwijk FJ, Sauter C, Hoedlmoser K, et al. The effect of daytime napping and full-night sleep on the consolidation of declarative and procedural information. J Sleep Res. 2019;28(1):1-9. doi:10.1111/jsr.12649

63. Fogel SM, Ray LB, Binnie L, Owen AM. How to become an expert: a new perspective on the role of sleep in the mastery of procedural skills. Neurobiol Learn Mem. 2015;125(1):236-248. doi:10.1016/j. nlm.2015.10.004

64. Morin A, Doyon J, Dostie V, et al. Motor sequence learning increases sleep spindles and fast frequencies in post-training sleep. Sleep. 2008;31(8):1149-1156.
65. Nishida M, Walker MP. Daytime naps, motor memory consolidation and regionally specific sleep spindles. Miall C, ed. PLoS One. 2007;2 (4):e341. doi:10.1371/journal.pone.0000341

66. Walker M, Brakefield T, Morgan A, Hobson JA, Stickgold R. Practice with sleep makes perfect: sleep-dependent motor skill learning. Neuron. 2002;35(1):205-211. doi:10.1016/S0896-6273(02) 00746-8

67. Clemens Z, Fabó D, Halász P. Overnight verbal memory retention correlates with the number of sleep spindles. Neuroscience. 2005;132 (2):529-535. doi:10.1016/j.neuroscience.2005.01.011

68. Gais S, Mölle M, Helms K, Born J. Learning-dependent increases in sleep spindle density. $J$ Neurosci. 2002;22(15):6830-6834. doi:10.1523/JNEUROSCI.22-15-06830.2002

69. Marshall L, Helgadóttir H, Mölle M, Born J. Boosting slow oscillations during sleep potentiates memory. Nature. 2006;444 (7119):610-613. doi:10.1038/nature05278

70. Lu W, Göder R. Does abnormal non-rapid eye movement sleep impair declarative memory consolidation? Disturbed thalamic functions in sleep and memory processing. Sleep Med Rev. 2012;16 (4):389-394. doi:10.1016/j.smrv.2011.08.001

71. Smith CT, Fazekas A. Amounts of REM sleep and stage 2 required for efficient learning. Sleep Res. 1997;26:690.

72. Conway JM, Smith CT. REM sleep and learning in humans: a sensitivity to specific types of learning tasks. J Sleep Res. 1994;3 (1):48.

73. Stawski RS, Sliwinski MJ, Almeida DM, Smyth JM. Reported exposure and emotional reactivity to daily stressors: the roles of adult age and global perceived stress. Psychol Aging. 2008;23(1):52-61. doi:10.1037/0882-7974.23.1.52

74. Callaway CW, Lydic R, Baghdoyan HA, Hobson JA. Pontogeniculooccipital waves: spontaneous visual system activity during rapid eye movement sleep. Cell Mol Neurobiol. 1987;7 (2):105-149. doi:10.1007/BF00711551

75. Brooks DC. Waves associated with eye movement in the awake and sleeping cat. Electroencephalogr Clin Neurophysiol. 1968;24 (6):532-541. doi:10.1016/0013-4694(68)90042-4

76. De Koninck J. Intensive learning, REM sleep and REM sleep mentation. Sleep Res Bull. 1995;1(2):39-40.

77. Hertenstein E, Gabryelska A, Spiegelhalder K, et al. Reference data for polysomnography-measured and subjective sleep in healthy adults. J Clin Sleep Med. 2018;14(4):523-532. doi:10.5664/ jesm.7036

78. McGregor KK, Alper RM. Sleep disorders as a risk to language learning and use. EBP Briefs. 2015;10(1):1-21.
Nature and Science of Sleep

\section{Publish your work in this journal}

Nature and Science of Sleep is an international, peer-reviewed, open access journal covering all aspects of sleep science and sleep medicine, including the neurophysiology and functions of sleep, the genetics of sleep, sleep and society, biological rhythms, dreaming, sleep disorders and therapy, and strategies to optimize healthy sleep.
The manuscript management system is completely online and includes a very quick and fair peer-review system, which is all easy to use. Visit http://www.dovepress.com/testimonials.php to read real quotes from published authors. 